\title{
Intractable Progressive Cerebral Infarction with Multiple Atypical Aneurysms in Systemic Lupus Erythematosus
}

\author{
Jongmin Lee, MD* (D); Hyun Young Kim, MD, PhD* (D); Young Seo Kim, MD, PhD* (D); \\ Sang-Cheol Bae, MD, $\mathrm{PhD}^{\dagger}$ (D); Ji Young Lee, MD, $\mathrm{PhD}^{\ddagger}$ (1); Young-Jun Lee, $\mathrm{MD}, \mathrm{PhD}^{\ddagger}$ (D) \\ Departments of Neurology ${ }^{\star}$, Rheumatology $^{\dagger}$, and Radiology ${ }^{\ddagger}$, Hanyang University College of Medicine, Seoul, Korea
}

\begin{abstract}
We report a case of intractable progressive cerebral infarction with multiple fusiform aneurysms in a 34-year-old female patient with systemic lupus erythematosus (SLE), non-responsive to massive immunotherapy. The patient visited the emergency department with dysarthria and left-sided hemiparesis that occurred 2 days before. She was diagnosed with SLE involving the brain and received 12 cycles of cyclophosphamide 12 years prior. Brain diffusion-weighted imaging showed acute infarctions involving the pons and medulla. Additionally, multifocal microbleeding-like signals in various cisternal spaces were detected using susceptibility-weighted imaging. Digital subtraction angiography revealed multiple fusiform aneurysms. Despite antithrombotic treatment with triflusal and immunotherapies, including corticosteroids, mycophenolate mofetil, and immunoglobulins, for cerebral vasculitis associated infarction, her neurologic deficits worsened with recurrent cerebral infarction. Further investigation for accurate diagnosis and treatment is required.
\end{abstract}

J Neurosonol Neuroimag 2021;13(2):71-75

Key Words: Cerebral infarction; Intracranial aneurysm; Lupus erythematosus, systemic
Received: October 27, 2021

Revised: November 14, 2021

Accepted: November 19, 2021

Correspondence:

Hyun Young Kim, MD, PhD

Department of Neurology,

Hanyang University College of

Medicine, 222 Wangsimni-ro,

Seongdong-gu, Seoul 04763 ,

Korea

Tel: $+82-2-2290-8367$

Fax: +82-2-2299-2391

E-mail: hyoungkim1@

hanyang.ac.kr
Systemic lupus erythematosus (SLE) is a rare etiology of stroke in young patients. The relative risk for ischemic and hemorrhagic stroke in SLE is two- and threefolds higher, respectively, than those in the general population. ${ }^{1,2}$ The neuropathologic findings of neuropsychiatric SLE (NPSLE) show the highest incidence of non-vasculitic vasculopathy of small arterioles and capillaries; however, cerebral vasculitis involving small vessels, including arteries and veins, is relatively low with an incidence of $7-13 \% .{ }^{3,4}$ The SLE-associated vasculitis induces cerebrovascular events, including rare forms of intracranial aneurysms and subarachnoid hemorrhage. ${ }^{5}$ Herein, we report a case of intractable, progressive cerebral infarction in an SLE patient presenting evidence of vasculitis involving cerebral arteries with multiple cerebral aneurysms and suspicious vasculitis involving cerebral veins.

\section{CASE}

A 34-year-old female experienced left facial palsy, horizontal diplopia, and unstable gait 1 day before the hospital visit. The initial National Institute of Health Stroke Scale (NIHSS) score was 6 (best gaze, 1; facial palsy, 1; right arm, 1; right leg, 1; left leg, 1; and dysarthria, 1). She was diagnosed with SLE 16 years prior and was currently treated with prednisolone (10 mg/day) and hydroxychloroquine (100 mg/day). Brain diffusion-weighted imaging (DWI) revealed multiple acute infarctions in the medulla and pons (Fig. 1A). Brain susceptibility-weighted imaging (SWI) displayed multifocal low signals in the ambient, quadrigeminal, basal cistern, and anterior surfaces of the brainstem (Fig. 1B). Brain magnetic resonance angiography revealed multiple narrowing and aneurysmal lesions in both anterior and 
posterior circulation arteries (Fig. $1 \mathrm{C}$ ). Cerebrospinal fluid (CSF) examination showed normal opening pressure (120 mmHg), colorless clear fluid, normal white (o/ $\left.\mathrm{mm}^{3}\right)$ and red $\left(1 / \mathrm{mm}^{3}\right)$ blood cell counts with increased protein $(98 \mathrm{mg} / \mathrm{dL})$ and normal glucose $(65 \mathrm{mg} / \mathrm{dL})$ levels compared with serum glucose level (123 mg/dL). Laboratory findings revealed normal white blood cell count $\left(9,300 / \mathrm{mm}^{3}\right)$, normal platelet count $\left(186,000 / \mathrm{mm}^{3}\right)$, normal erythrocyte sedimentation rate $(6 \mathrm{~mm} /$ hour$)$, increased C-reactive protein
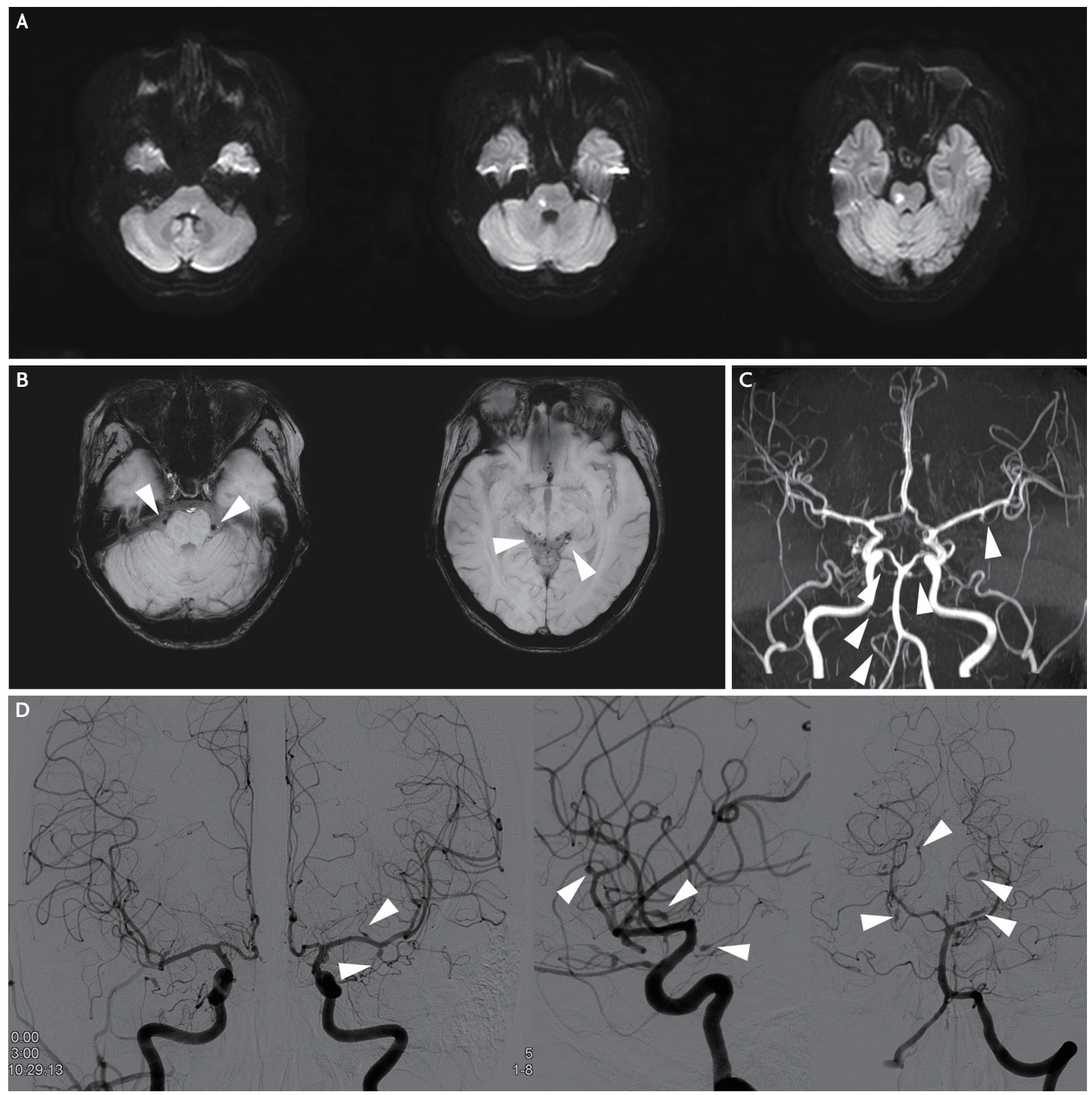

FIG. 1. Brain magnetic resonance imaging and angiogram of the patient. (A) Brain diffusion-weighted imaging at the first visit shows multiple acute infarctions in the medulla and pons. (B) Brain susceptibility-weighted imaging shows multifocal microbleed-like low signals (arrowheads) in basal and quadrigeminal cisterns. (C) Brain magnetic resonance angiography shows multiple narrowing and dilatation of intracranial arteries (arrowheads). (D) Digital subtraction angiography shows multiple fusiform aneurysms in intracranial arteries in both anterior and posterior circulation (arrowheads). 

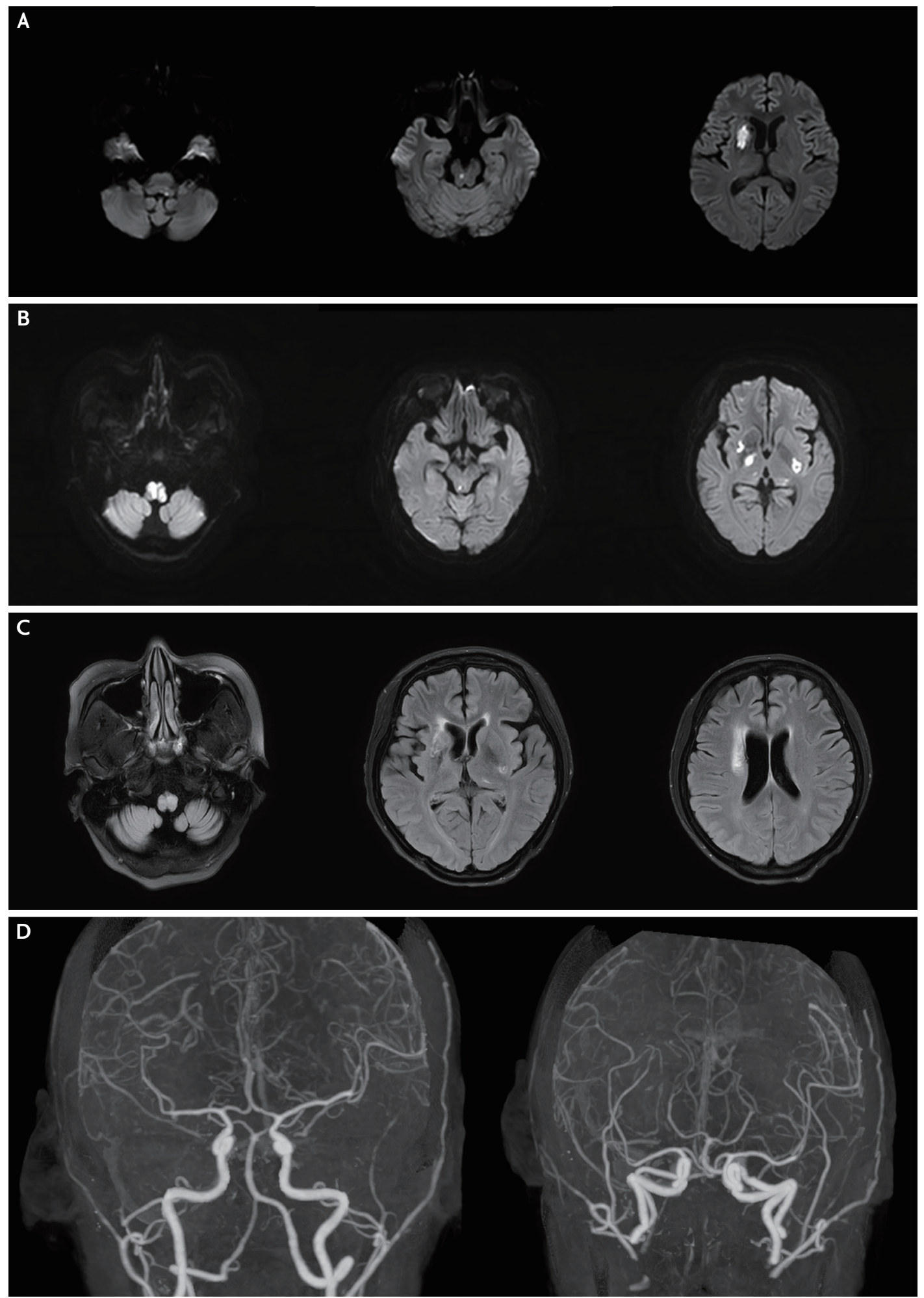

FIG. 2. Follow-up brain magnetic resonance imaging (MRI) findings of the patient. (A) Diffusion-weighted imaging (DWI) at second admission shows newly-developed infarction in the medulla, pons, and right basal ganglia. (B) On day 5 following the second admission, DWI after intravenous cyclophosphamide and high dose methylprednisolone administration shows newly developed acute infarction on both right and left medulla and basal ganglia and right midbrain. (C) Follow-up MRI after 1 year. Fluid attenuated inversion recovery imaging shows encephalomalacic changes in the medulla, right and left basal ganglia, and right corona radiata. (D) Computed tomographic angiography after 1 year shows improved intracranial stenosis and aneurysms. 
( $0.707 \mathrm{mg} / \mathrm{dL})$, negative antiphospholipid antibodies, positive anti-dsDNA antibody (1:115), and decreased complement level $\left(\mathrm{C}_{3}, 21 \mathrm{mg} / \mathrm{dL} ; \mathrm{C}_{4}, 8 \mathrm{mg} / \mathrm{dL}\right)$, representing rather high disease activity. Based on imaging and laboratory findings, we diagnosed acute ischemic stroke due to SLE-associated vasculitis and prescribed triflusal (600 mg/day), mycophenolate mofetil (1 g/day), increased prednisolone dose (40 mg/day), and hydroxychloroquine (200 mg/day). Despite evidence of no bleeding in the CSF study, the microbleed-like signals on SWI and multiple aneurysms restricted to the high potency antithrombotics use. Digital subtraction angiography performed 3 days later showed more prominent multiple atypical fusiform aneurysms and stenotic lesions in the anterior and posterior circulation arteries (Fig. 1D). The patient was discharged 10 days after admission with improved neurological symptoms and an NIHSS score of 2 (facial palsy, 1; dysarthria, 1).

After 1 month, she manifested newly developed dysarthria and left hemiparesis. Her NIHSS score was 4 (facial palsy, 1; left arm, 1; left leg, 1; dysarthria, 1) at the second admission. DWI revealed acute infarctions in the right basal ganglia, right midbrain, and left medulla (Fig. 2A). Despite the increasing dose of methylprednisolone (625 mg/day), and intravenous cyclophosphamide (500 mg/day) and immunoglobulin ( $2 \mathrm{~g} / \mathrm{kg}$ ) administration, her neurologic deficits worsened to drowsiness, respiratory difficulty, and quadriplegia, resulting in an NIHSS score of 20 (facial palsy, 1; quadriplegia, 16; sensory, 2; dysarthria, 1). Follow-up DWI showed newly developed acute infarctions in both medulla, basal ganglia and right midbrain (Fig. 2B). Despite massive immunotherapies, her neurological symptoms stabilized without improvement.

At 1 year follow-up, neurologic symptoms, including quadriparesis (MRC grade 2), dysphagia, and diplopia, remained. Brain DWI displayed no acute lesions; however, fluid-attenuated inversion recovery imaging showed encephalomalacic changes in the bilateral medulla and right basal ganglia (Fig. 2 C). Although computed tomographic angiography can underestimate small arterial lesions, intracranial stenosis and aneurysms showed improvement compared to previous images (Fig. 2D).

\section{DISCUSSION}

This case involved a woman with SLE who presented with cerebral vasculitis, multiple intracranial fusiform aneurysms, and recurrent intractable ischemic strokes. Although we used known therapeutic algorithms for NPSLE, ${ }^{6}$ we could not stop the progression of the disease and severe neurologic sequelae in the patient.

The cerebral aneurysm incidence rate in SLE patients is relatively low. Nevertheless, SLE-associated cerebral aneurysms can grow rapidly and consequently rupture, entailing an increased mortality rate. ${ }^{7}$ Furthermore, cerebral aneurysms in SLE patients are mostly localized to the distal branches, forming fusiform and saccular aneurysms. ${ }^{8}$ Therefore, SLE-related cerebral aneurysms are difficult to treat. The aneurysm formation mechanism is still unclear, but transmural angiitis has been suggested using postmortem histopathological studies. ${ }^{9}$ In this case, cerebral vasculitis might have caused recurrent ischemic strokes and cerebral aneurysms. However, the treatment was challenging because of her hemorrhagic risk.

Cerebrovascular disease as a form of NPSLE has been reported, and no standard or therapeutic strategies have been established. The current recommendation classifies ischemic NPSLE into cerebral vasculitis and atherothrombotic infarction and suggests immunotherapy and antiplatelet or anticoagulation therapy, respectively., ${ }^{3,6,10}$ Given the autoimmune inflammatory mechanism of cerebral vasculitis, high-dose corticosteroids, cyclophosphamide, mycophenolate mofetil, immunoglobulins, and rituximab have been suggested. ${ }^{6}$ Rituximab was not used in this case due to the permanent neurologic damage risk; however, the other immunotherapies failed to reduce the disease activities. Unfortunately, a more potent antithrombotic therapy could not be used. The main limitation in the antithrombotic therapy selection was the co-existence of multiple ischemic strokes, microbleed-like signals, and multiple fusiform aneurysms. The risk of intracranial hemorrhage prevented the use of more potent antithrombotics, such as heparin, low-molecular-weight heparin, and dual antiplatelets. However, reconsidering the CSF examination findings, which revealed no hemorrhage incidence, the bleeding risk due to microbleed-like signals in the cisterns was low. The low 
signals, which disappeared after 1 year, might represent venous vasculitis.

Previous studies on stroke in SLE have used the arbitrary and composite stroke classification system: any stroke, ischemic stroke, and intracerebral hemorrhage. ${ }^{1}$ However, SLE-associated stroke may have a different mechanism from conventional risk factor-related stroke, and more detailed diagnosis and treatment are needed. Moreover, future randomized clinical trials using antithrombotics and combined immune therapy are essential for establishing standard treatment guidelines.

\section{Ethics Statement}

This study was approved by the Institutional Review Board (IRB) of Hanyang University Hospital (IRB No. 2021-10-037). Informed participation consent was waived by the Review Board.

\section{Availability of Data and Material}

Due to the nature of this case report, patient did not agree for her data to be shared publicly, so supporting data is not available.

\section{Acknowledgments}

None.

\section{Sources of Funding}

None.

\section{Conflicts of Interest}

No potential conflicts of interest relevant to this article was reported.

\section{REFERENCES}

1. Holmqvist M, Simard JF, Asplund K, Arkema EV. Stroke in systemic lupus erythematosus: a meta-analysis of population-based cohort studies. RMD Open. 2015;1:e000168.

2. Arkema EV, Svenungsson E, Von Euler M, Sjöwall C, Simard JF. Stroke in systemic lupus erythematosus: a Swedish population-based cohort study. Ann Rheum Dis. 2017;76:15441549.

3. Carrión-Barberà I, Salman-Monte TC, Vílchez-Oya F, Monfort J. Neuropsychiatric involvement in systemic lupus erythematosus: a review. Autoimmun Rev. 2021;20:102780.

4. Scolding NJ. Central nervous system vasculitis. Semin Immunopathol. 2009;31:527-536.

5. Torné R, Rodríguez-Hernández A, Bernard T, Arikan Abelló F, Vilalta Castan J, Sahuquillo J. Subarachnoid hemorrhage in systemic lupus erythematosus: systematic review and report of three cases. Clin Neurol Neurosurg. 2015;128:17-24.

6. Magro-Checa C, Zirkzee EJ, Huizinga TW, Steup-Beekman GM. Management of neuropsychiatric systemic lupus erythematosus: current approaches and future perspectives. Drugs. 2016;76:459-483.

7. Graffeo CS, Tanweer O, Nieves CF, Belmont HM, Izmirly PM, Becske T, et al. Rapid aneurysm growth and rupture in systemic lupus erythematosus. Surg Neurol Int. 2015;6:9.

8. Majidi S, Leon Guerrero CR, Gandhy S, Burger KM, Sigounas D. Numerous fusiform and saccular cerebral aneurysms in central nervous system lupus presenting with ischemic stroke. J Stroke Cerebrovasc Dis. 2017;26:e126-e128.

9. Kelley RE, Stokes N, Reyes P, Harik SI. Cerebral transmural angiitis and ruptured aneurysm: a complication of systemic lupus erythematosus. Arch Neurol. 1980;37:526-527.

10. Nikolopoulos D, Fanouriakis A, Bertsias G. Treatment of neuropsychiatric systemic lupus erythematosus: clinical challenges and future perspectives. Expert Rev Clin Immunol. 2021;17:317-330. 